\title{
avß6 integrin may be a potential prognostic biomarker in interstitial lung disease
}

\author{
Gauri Saini ${ }^{1}$, Joanne Porte ${ }^{1}$, Paul H. Weinreb ${ }^{2}$, Shelia M. Violette ${ }^{2}$, \\ William A. Wallace ${ }^{3}$, Tricia M. McKeever ${ }^{4}$ and Gisli Jenkins ${ }^{1}$ \\ Affiliations: 'Division of Respiratory Medicine, University of Nottingham, Nottingham, UK. ${ }^{2}$ Biogen Idec, \\ Cambridge, MA, USA. ${ }^{3}$ Division of Pathology, University of Edinburgh, Edinburgh, UK. ${ }^{4}$ Division of \\ Epidemiology and Public Health, University of Nottingham, Nottingham, UK.
}

Correspondence: Gauri Saini, Division of Respiratory Medicine, University of Nottingham, B-35, Clinical Sciences Building, Nottingham City Hospital, Hucknall Road, Nottingham, NG15 9EY, UK.

E-mail: gauri.sainianottingham.ac.uk

ABSTRACT Idiopathic pulmonary fibrosis (IPF) and fibrotic nonspecific interstitial pneumonitis are progressive interstitial lung diseases (ILDs) with limited treatment options and poor survival. However, the rate of disease progression is variable, implying there may be different endotypes of disease. We hypothesised that immunophenotyping biopsies from ILD patients might reveal distinct endotypes of progressive fibrotic disease, which may facilitate stratification when undertaking clinical trials of novel therapies for IPF.

43 paraffin-embedded, formalin-fixed lung tissue sections were immunostained for five molecules implicated in the pathogenesis of the fibrosis: $\alpha$-smooth muscle actin ( $\alpha \mathrm{SMA}), \alpha \mathrm{v} \beta 6$ integrin, pro-surfactant protein C (SP-C), hepatocyte growth factor (HGF) and tenascin-C (TenC). Levels of immunostaining and numbers of fibroblastic foci were quantified using operator-dependent and -independent methods. The relationship of all these markers to overall survival was analysed.

Staining revealed high levels of $\alpha \mathrm{SMA}, \alpha v \beta 6$ integrin, pro-SP-C, HGF and TenC, and fibroblastic foci. Immunostaining varied across samples for all molecules but only the extent of $\alpha v \beta 6$ integrin immunostaining was associated with increased mortality. There was no association with the other markers measured.

Our data suggest high levels of $\alpha v \beta 6$ integrin may identify a specific endotype of progressive fibrotic lung disease.

@ERSpublications

High levels of $\alpha \mathrm{v} \beta 6$ integrin immunostaining on VATS biopsy predict worse survival than low levels of immunostaining http://ow.ly/IyCfH

This article has supplementary material available from erj.ersjournals.com

Received: May 082014 | Accepted after revision: Jan 16 2015 | First published online: March 052015

Support statement: This study was supported by the UK Medical Research Council (MRC Industrial Collaboration Agreement G0901226). Funding information for this article has been deposited with FundRef.

Conflict of interest: Disclosures can be found alongside the online version of this article at erj.ersjournals.com

Copyright OERS 2015 


\section{Introduction}

Fibrotic interstitial lung diseases (ILDs), including idiopathic pulmonary fibrosis (IPF) and fibrotic nonspecific interstitial pneumonitis (NSIP) are chronic, progressive diseases associated with serious morbidity and premature death. The median survival for IPF is $\sim 3$ years [1]. Currently, 4500 new cases of IPF arise each year in the UK, and both the incidence and mortality have been rising for the last few decades [2]. NSIP is considered a distinct clinical entity, with a better prognosis compared with IPF, although many patients suffer from progressive disease [3]. Furthermore, there is a large group of patients with unclassifiable ILD with a similar progressive, but heterogeneous, clinical course [4]. The heterogeneous nature of progressive fibrotic ILDs suggests there may be endotypes of disease, both distinct and common, across the different clinical phenotypes.

There is considerable interest in defining endotypes of progressive fibrotic ILD both to understand disease progression and to facilitate stratified treatment design. Transcriptional profiling is able to distinguish patients with stable disease from those who have progressive disease [5] and acute exacerbations [6], although no difference in mortality was documented. Studies of biopsies from patients with IPF have assessed whether the fibroblastic foci correlate with reduced survival but the results have not been conclusive $[7,8]$, and molecular phenotyping for transforming growth factor (TGF)- $\beta$ signalling molecules did not show any relationship to survival [9]. Serum and plasma biomarkers, such as KL-6 [10], CXCL13 [11] CCL18 [12], matrix metalloproteinase (MMP)-3 [13] and MMP-7 [14], have been shown to have prognostic value in IPF, although how they reflect underlying disease pathogenesis or whether they change in response to therapy remains uncertain. Recently, it was hypothesised that there may be two distinct endotypes of disease, related to disrupted bronchiolisation and lymphoid aggregates, which may prove useful in developing interventional clinical trials [13].

IPF is thought to result from initial injury of alveolar epithelium leading to activation of lung fibroblasts, which subsequently synthesise excess matrix. The $\alpha v \beta 6$ integrin is an epithelial restricted molecule that has been implicated in numerous models of lung fibrosis [15-18] and in patients with IPF [16, 18], and targeted therapy is currently undergoing phase 2 evaluation in the USA (www.clinicaltrials.gov identifier number NCT01371305). Surfactant protein C (SP-C) is another epithelium-specific protein that has been associated with IPF. SP-C levels are increased in IPF bronchoalveolar lavage [19], and genetic studies have implicated SP-C gene mutations in both sporadic and familial IPF [20]. The emergence of a myofibroblast phenotype, characterised by $\alpha$-smooth muscle actin ( $\alpha$ SMA) expression, is a key event in the pathogenesis of fibrosis and may be responsible for the increased contractility in tissues undergoing fibrosis [21]. Hepatocyte growth factor (HGF) is increased in response to tissue injury, and inverse correlations have been identified for HGF expression during the development and progression of fibrosis in several tissues, including the lung [22]. Furthermore, lung fibroblasts isolated from IPF patients have decreased HGF expression and activation relative to fibroblasts from control patients [23]. Tenascin-C (TenC) is increased in fibrosis, especially in usual interstitial pneumonitis (UIP), a hallmark of IPF [24]. It is abundant in the fibroblast foci of active fibrosis and is also present in the basement membrane regions beneath the metaplastic epithelium lining honeycomb cysts [25].

We evaluated these molecules by immunohistochemical analysis of fibrotic ILD tissue, as well as quantifying fibroblastic foci, to determine whether specific epithelial, or mesenchymal, endotypes of progressive fibrotic lung disease exist to facilitate clinical trial stratification.

\section{Methods}

\section{Reagents}

Xylene and 100\% ethanol were purchased from Fisher Scientific (Loughborough, UK). 30\% hydrogen peroxide, methanol, $10 \mathrm{mM}$ sodium citrate buffer, horse serum, goat serum, Trizma base, SIGMAFAST 3,3'-diaminobenzidine (DAB) tablets, Mayer's haematoxylin and eosin were purchased from Sigma-Aldrich (Poole, UK). The commercially available ABC kit was used from Vectastain Universal ABC Kit (Vector Laboratories, Peterborough, UK). The antibodies used were anti- $\alpha$ SMA mouse monoclonal (clone 1A4; Abcam, Cambridge, UK), TenC mouse monoclonal (NCL-TENAS C; Novocastra, Newcastle upon Tyne, UK), anti-c-Met mouse monoclonal (Invitrogen, Paisley, UK) and anti-pro-SP-C rabbit polyclonal (Millipore, Watford, UK) were used. The anti- $\alpha v \beta 6$ integrin antibody (6.2A1; Biogen Idec, Cambridge, MA, USA) was generated as previously described [26]. The secondary antibodies used were biotinylated horse anti-mouse, biotinylated goat anti-mouse and biotinylated goat anti-rabbit (Vector Laboratories).

\section{Patient samples}

43 ILD lung sections were obtained via video-assisted thoracoscopic surgery (VATS) biopsy, which were formalin fixed and paraffin embedded. The sections were obtained from W. Wallace (University of Edinburgh, Edinburgh, UK). These patients were seen in an outpatient clinic in Edinburgh from 1999 to 
2009 and required biopsy for diagnostic purposes. All patients had a diagnosis of IPF or NSIP as per the international consensus criteria [27]. They were subsequently followed over a 10-year period to allow analysis of "all cause" mortality; unfortunately, there are insufficient clinical data to determine the cause of death. The tissue samples were obtained after informed consent and local ethics approval (South East Scotland SAHSC Bioresource 06/S1101/41).

\section{Immunohistochemistry}

Parallel lung tissue sections were cut and deparaffinised in xylene followed by rehydration in decreasing concentrations of ethanol, and washed in Tris-buffered saline. Antigen retrieval, and the primary and secondary antibody for each marker are listed in table S1. Endogenous peroxidase activity was blocked using $3 \%$ hydrogen peroxide in methanol for $30 \mathrm{~min}$. The sections were washed twice with Tris-buffered saline and incubated with a blocking buffer of $5 \%$ goat or horse serum, for $20 \mathrm{~min}$ at room temperature. Sections were incubated with primary antibody for $1 \mathrm{~h}$ at room temperature or overnight at $4^{\circ} \mathrm{C}$. Subsequently, sections were washed with Tris-buffered saline and incubated for $1 \mathrm{~h}$ with a biotin-conjugated secondary antibody. Signal amplification was performed using the Vectastain standard ABC kit prior to visualisation with DAB for $10 \mathrm{~min}$. Slides were counter stained in Mayer's haematoxylin (Sigma-Aldrich). Secondary antibody only controls were used for all experiments.

\section{Quantification analysis}

Each patient's sample was analysed for two epithelial markers ( $\alpha \mathrm{v} \beta 6$ integrin and SP-C) and three mesenchymal markers ( $\alpha \mathrm{SMA}$, HGF and TenC); therefore, 43 samples were analysed for five immunomarkers. The interpretation of histological sections is an inherently subjective process based primarily on morphological features. When used by an experienced operator, the scoring methods have been shown to have statistically significant correlations with clinical outcomes [28]. Automated analysis has been increasingly used in recent years to attempt to standardise quantification. In the absence of a clear consensus on the most accurate quantification method, two techniques incorporating both semiquantitative and automated analysis were used.

\section{Operator-dependent, semiquantitative analysis}

Individual patient slides contained between one and five lung sections. Five high-power fields (HPFs) per tissue section (maximum of 25 per slide) were analysed. To normalise for varying numbers of regions of interest per slide, the mean score per slide was calculated. For epithelial markers, the degree of staining is expressed as the percentage of the epithelial surface in the selected HPF. For the purpose of quantification, it was assumed that all cells on the luminal surface were epithelial in origin. For the mesenchymal markers, the whole section was taken into account for analysis and the degree of staining is expressed as a percentage of the whole section. To avoid overlapping analysis of individual tissue sections, the slides were viewed in a structured manner from right to left, both when analysing each tissue section and each slide. The coding was performed prior to analysis as: 0 , negative; $1,0-\leqslant 30 \% ; 2,30-\leqslant 50 \% ; 3,50-\leqslant 70 \%$; and 4 , $\geqslant 70 \%$. Each slide was analysed by two independent investigators blinded to the outcome of the patient. Weighted $\kappa$-analysis was performed to measure agreement between two operators. In patients where there was a disagreement, an average of the two was used for analysis.

\section{Automated image analysis}

Automated image analysis using Nikon 90i microscope image analysis software (NIS Elements; Nikon UK Ltd, Kingston upon Thames, UK) was performed. A threshold using the red-green-blue (RGB) mode was selected to determine the pixels to be included in the section. The threshold limit was defined using reference points from within the image in the positive control. For epithelial surfaces, a single-point tool was used to define threshold, while a three-point circle was used for the mesenchymal markers. Using the Threshold ND image tool, the threshold was uniform for all frames. An algorithm to define a histological region of interest (ROI), to define signal localisation, was used. The ROI was selected at random and was fixed in terms of area. Five HPFs per section (maximum of 25 per slide) were taken for the purpose of analysis. The amount of stain is represented as percentage of the ROI area, with the average of the five HPFs as the numerator and the ROI area as the denominator.

\section{Fibroblastic focus quantification}

Fibroblastic focus quantification was performed as previously described [8]. The following criteria were used to identify fibroblastic foci based on previously published definitions [8, 29]: 1) discrete subepithelial areas of spindle shaped myofibroblasts and fibroblasts; 2) linear arrangement of cells parallel to luminal surface; and 3) pale staining matrix in contrast with darker parenchyma. Using a 10× objective lens, five fields per tissue section (maximum of 25 per slide) were analysed. Each slide was analysed by two independent observers and mean score of the two was used for analysis. 
TABLE 1 Demographics of 43 interstitial lung disease patients undergoing video-assisted thoracoscopic surgery biopsy in Edinburgh, UK (1999-2009)

\section{Age years \\ Males/females n $(\%)$ \\ Diagnosis UIP/NSIP\# $n$ \\ Survival after biopsy months"}

$63(43-76)$

$24 / 19(56 / 44)$

$36 / 5$

$68(11-115)$

Data are presented as median (range), unless otherwise stated. UIP: usual interstitial pneumonitis; NSIP: nonspecific interstitial pneumonitis. ": two patients has unclassifiable fibrotic interstitial lung disease; ${ }^{\natural}: \mathrm{n}=43$.

\section{Statistics}

The start date was the date of the biopsy. The end date was taken as the date of death or the last known date the patient was alive (November 15, 2011). Patients were censored on the last known date that they were alive (November 15, 2011). There is no gold standard for immunohistochemical analyses; hence, the groups were divided into quartiles for operator-dependent and tertiles for automated analysis. Ranked analysis was performed and p-values for trend (ptrend) are shown. Cox's proportional hazards regression and Kaplan-Meier survival analysis were carried out. The quantification was performed blind to the survival data. Spearman correlation was performed between the operator-dependent and automated analyses. Stata Statistical Software, release 11, (StataCorp LP, College Station, TX, USA) was used.

\section{Results}

\section{Patient demographics}

Basic demographic data are shown in table 1. The median age of patients was 63 years, with a slight predominance of males. 36 patients had UIP and five had NSIP.
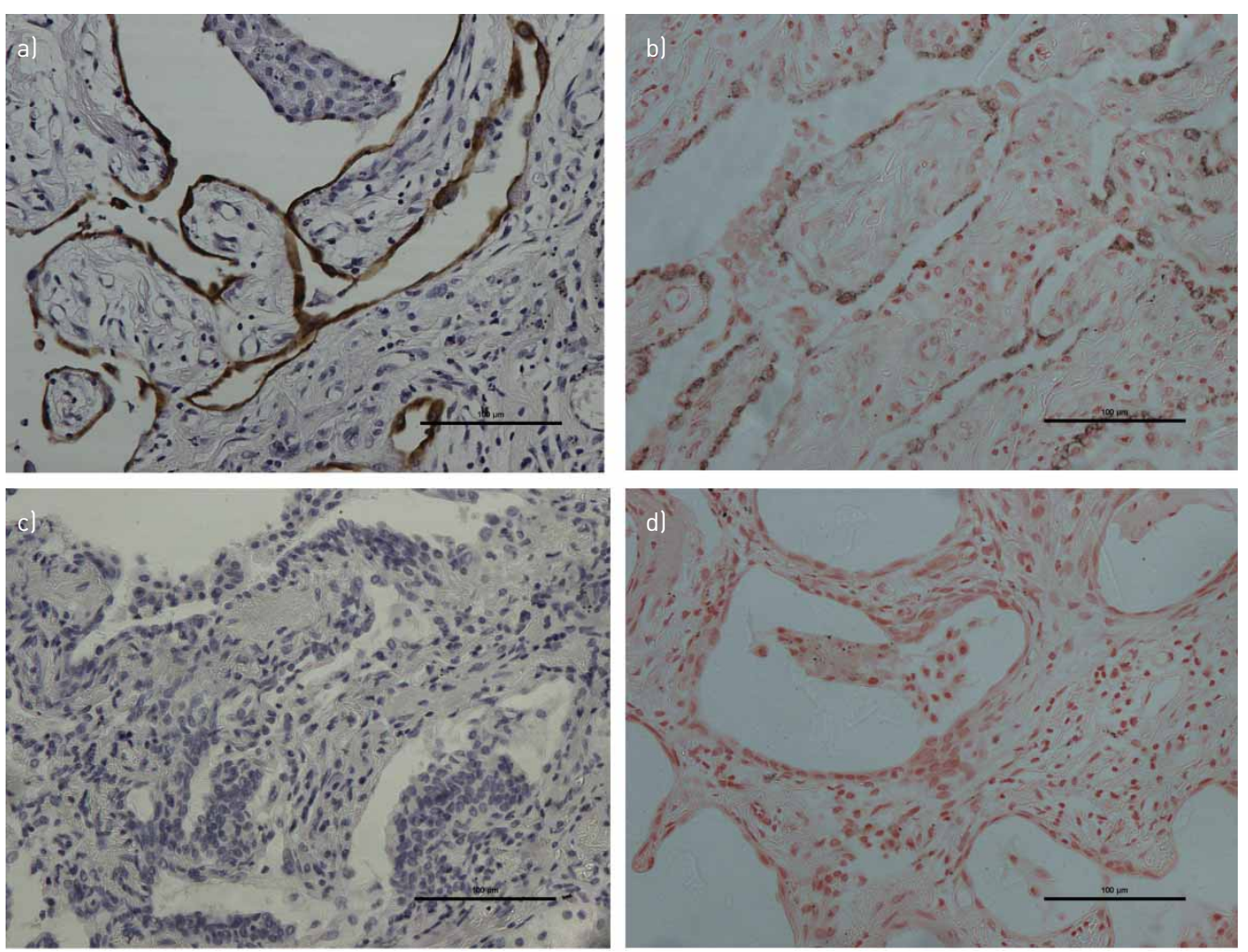

FIGURE 1 Immunohistochemical staining in paraffin-embedded, formalin-fixed surgical lung biopsies from 43 fibrotic interstitial lung disease patients. Immunostaining shows high levels of epithelial markers ( $\alpha$ v $\beta 6$ integrin and pro-surfactant protein C (SP-C)) compared with secondary antibody-only control. Representative sections of idiopathic pulmonary fibrosis lung are shown with control for each immunomarker. a) $\alpha v \beta 6$ integrin staining in the alveolar epithelial cells. b) pro-SP-C staining in the cytoplasm of the epithelial cell layer in a patchy distribution. c) $\alpha v \beta 6$ integrin secondary antibody control. d) pro-SP-C secondary antibody control. Scale bars $=100 \mu \mathrm{m}$. 
ILD patient biopsies stain positively for a range of immunomarkers

All sections stained positively for the five immunomarkers assessed when compared with secondary antibody controls (figs 1 and 2). $\alpha \mathrm{v} \beta 6$ integrin and pro-SP-C stained only in the epithelium (fig. 1a and b), consistent with their known cellular distribution. $\alpha$ SMA stained in the myofibroblasts in the mesenchymal regions of the fibrotic lung parenchyma (fig. 2a). HGF was expressed diffusely in the interstitium, excluding the epithelial surfaces (fig. 2c). TenC showed positive staining in the fibrotic interstitium and stromal fibres underlying the immune-negative epithelium (fig. 2b).

\section{Relationship between immunostaining and prognosis}

Weighted $\kappa$-agreement between the two investigators recording semiquantitative analysis was $81 \%$. Furthermore, the semiquantitative and automated analyses were strongly associated $\left(\mathrm{r}_{\mathrm{s}}=0.83, \mathrm{p}<0.0001\right)$. Of the five immunomarkers assessed, only $\alpha v \beta 6$ integrin was found to be associated with prognosis, with high levels of immunostaining being associated with a worse prognosis using both operator-dependent and automated image analysis methodology (table 2). There was a four-fold increased risk of death (95\% CI $0.87-20.23)$ in patients expressing the highest levels of $\alpha v \beta 6$ integrin using the operator-dependent analysis ( ptrend $=0.0019$ ) and two times increased risk of death (95\% CI 0.75-5.7) in high expressers of $\alpha v \beta 6$ integrin using the automated analysis (table 2). A worse median survival of 25 months was seen in patients with highest expression of $\alpha v \beta 6$ integrin (log rank equality test, $\mathrm{p}<0.0001$ ) (fig. 2). Differences in survival are shown in the Kaplan-Meier curves (fig. 3).

A similar trend was also seen with $\alpha \mathrm{SMA}$ using the operator-dependent analysis, with high levels of immunostaining being associated with shorter survival; however, these results did not reach statistical significance by either methodology (table 2).

There was no association between levels of immunostaining of HGF, TenC or pro-SP-C and prognosis by either methodology (table 2). The automated image analysis for pro-SP-C, using the RGB thresholding,
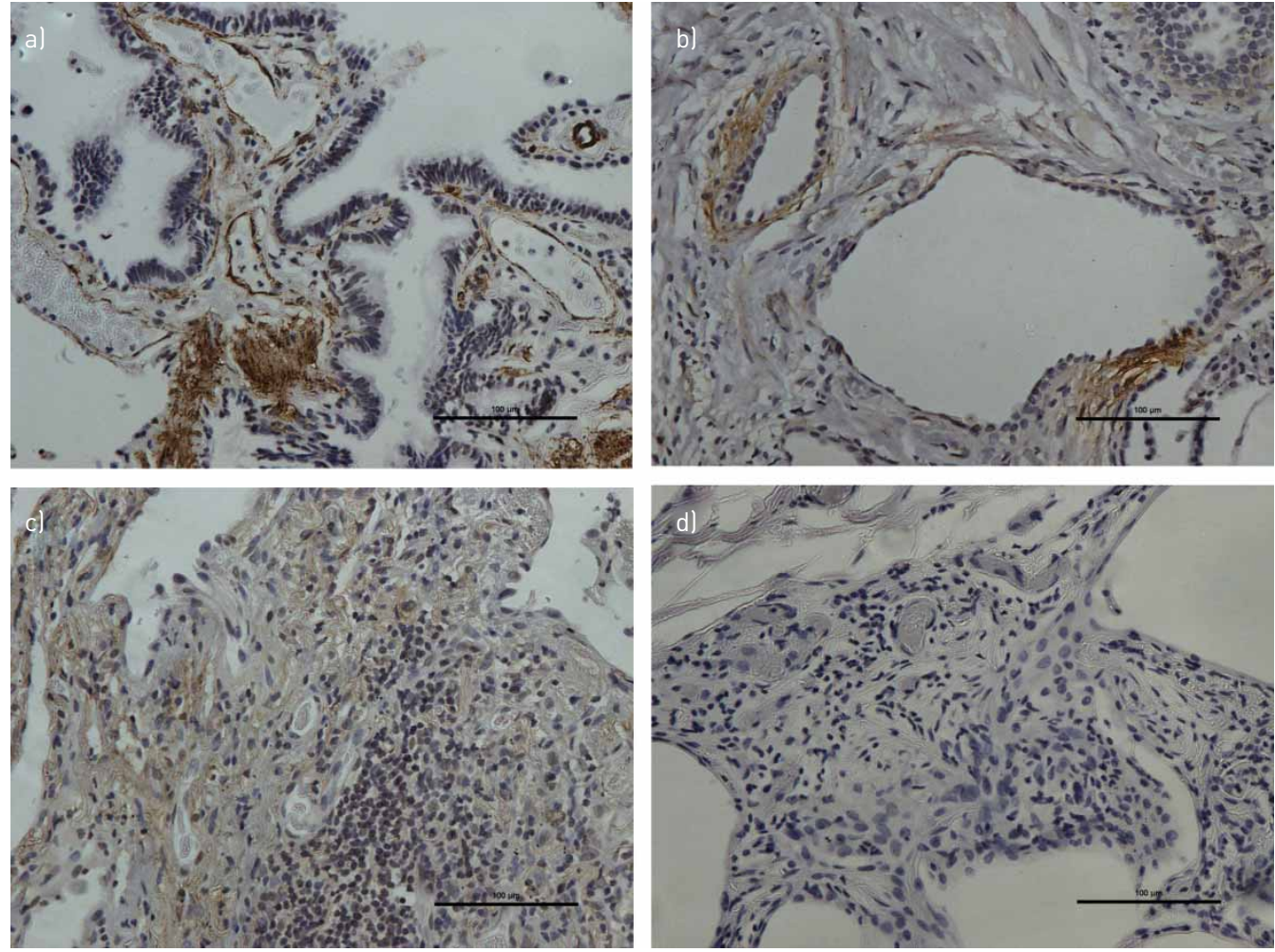

FIGURE 2 Immunohistochemical staining in paraffin-embedded, formalin-fixed surgical lung biopsies from 43 fibrotic interstitial lung disease patients. Immunostaining shows high levels of mesenchymal markers ( $\alpha$-smooth muscle actin $(\alpha S M A)$, hepatocyte growth factor (HGF) and tenascin-C (TenC)) compared with secondary antibody-only control. Representative sections of idiopathic pulmonary fibrosis lung are shown with control for each immunomarker. a) $\alpha$ SMA staining within the fibroblastic foci, showing cluster of cells composed of myofibroblasts. b) TenC staining in the stromal fibres beneath the immune-negative epithelial layer. c) HGF present diffusely in the interstitium along with negatively immune-stained alveolar epithelial cells, consistent with its known location. d) Secondary antibody controls for a-c. Scale bars $=100 \mu \mathrm{m}$. 
TABLE 2 Hazard ratios ${ }^{\#}$ for relationship between quantitative immunostaining and death in 43 interstitial lung disease patients, 1999-2009

\begin{tabular}{|c|c|c|c|c|}
\hline Biomarker & Method & Group (patients $\mathrm{n}$ ) & Hazard ratio $(95 \% \mathrm{CI})$ & $\mathrm{p}$-value for trend \\
\hline \multirow{4}{*}{$\alpha v \beta 6$} & & Group 2: $30-\leqslant 50 \%(14)$ & $0.71(0.134-3.80)$ & \\
\hline & & Group 4: >70\% (14) & $4.19(0.87-20.23)$ & \\
\hline & Automated & Group 1: low expression (15) & 1 & 0.25 \\
\hline & & Group 2: medium expression (14) & $0.98(0.32-3.06)$ & \\
\hline \multirow{6}{*}{ aSMA } & & Group 2: $30-\leqslant 50 \%(8)$ & $0.99(0.10-9.74)$ & \\
\hline & & Group 3: $50-\leqslant 70 \%(12)$ & $4.54(0.49-41.93)$ & \\
\hline & & Group 4: >70\% (21) & $1.77(0.22-14.01)$ & \\
\hline & Automated & Group 1: low expression (15) & 1 & 0.72 \\
\hline & & Group 2: medium expression (14) & $1.23(0.41-3.73)$ & \\
\hline & & Group 3: high expression (14) & $1.54(0.52-4.54)$ & \\
\hline \multirow{2}{*}{ HGF } & & Group 2: medium expression (14) & $1.70(0.65-4.48)$ & \\
\hline & & Group 3: high expression (14) & $0.84(0.28-2.52)$ & \\
\hline \multirow[t]{4}{*}{$S P-C^{\pi}$} & Semiquantitative & Group 1: 0-30\% (12) & 1 & 0.99 \\
\hline & & Group 2: $30-\leqslant 50 \%(14)$ & $1.17(0.37-3.70)$ & \\
\hline & & Group 3: $50-\leqslant 70 \%$ (9) & $1.25(0.32-4.78)$ & \\
\hline & & Group 4: $>70 \%(8)$ & $1(0.30-3.28)$ & \\
\hline \multirow[t]{4}{*}{ TenC $^{+}$} & Semiquantitative & & $1.16(0.36-1.97)$ & 0.58 \\
\hline & Automated & Group 1: low expression (15) & 1 & 0.57 \\
\hline & & Group 2: medium expression (14) & $1.62(0.55-4.83)$ & \\
\hline & & Group 3: high pressure (14) & $1.76(0.55-5.62)$ & \\
\hline
\end{tabular}

gave a falsely high positivity; thus, only operator-dependent results are shown. There was no difference in the results on excluding the unclassifiable biopsies.

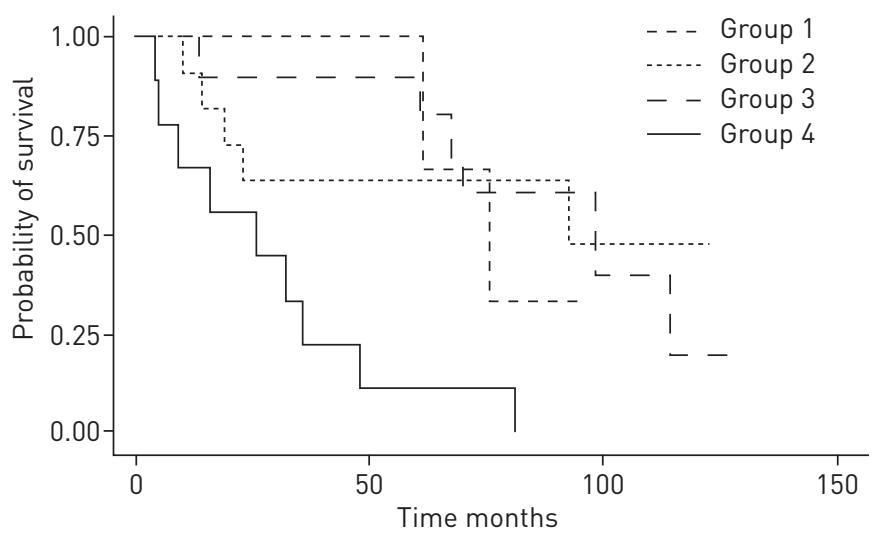

FIGURE 3 Kaplan-Meier survival for $\alpha \mathrm{v} \beta 6$ integrin immunomarker in 43 interstitial lung disease patients with video-assisted thoracoscopic surgery biopsy. Survival graph of $\alpha v \beta 6$ integrin expression, with the highest expressing group 4 versus groups 1, 2 and 3 . Median survival of groups 4 and $1-3$ was 25 and 92 months, respectively $(\mathrm{p}=0.0019)$. 

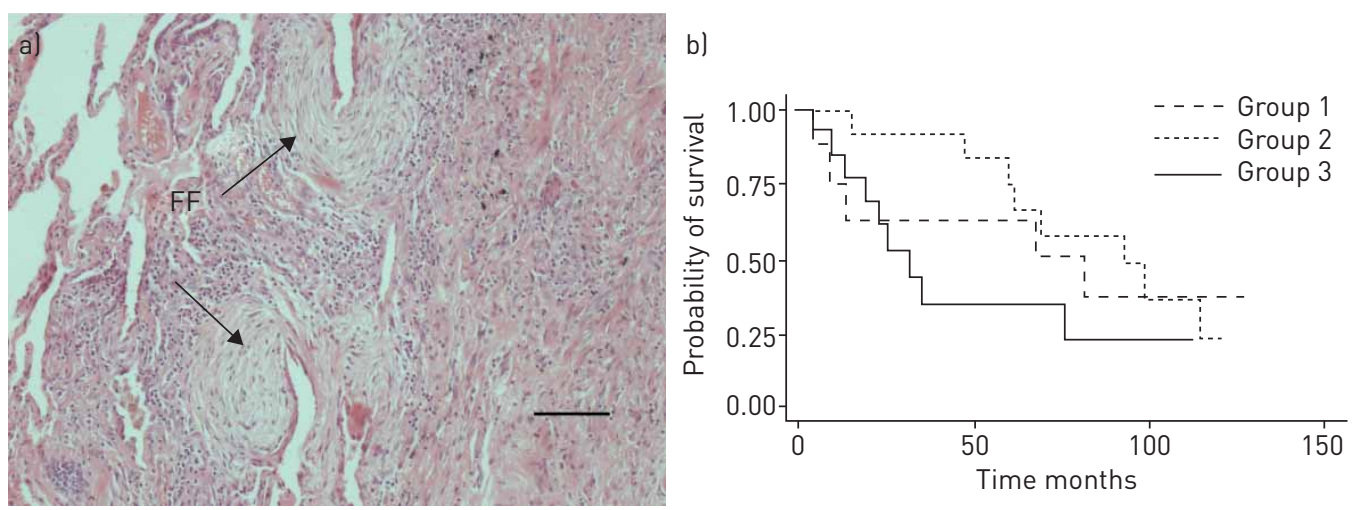

FIGURE 4 Assessment of fibroblastic foci (FF) in paraffin-embedded, formalin-fixed surgical lung biopsies from 43 fibrotic interstitial lung disease (ILD) patients. a) Section of lung tissue illustrating FF (arrows) using 10× objective lens. Scale bar $=100 \mu \mathrm{m}$. b) Kaplan-Meier survival for FF in 43 ILD patients following video-assisted thoracoscopic surgery biopsy. Group 1: $<1.3 \mathrm{FF}$ per section; group 2: 1.3-<3 FF per section; group 3: 3-<7 FF per section.

\section{Relationship between fibroblastic foci and prognosis}

Haematoxylin and eosin staining was used to identify fibroblastic foci (fig. 4a). Weighted $\kappa$-agreement between the two investigators recording fibroblastic foci was $68 \%$. Using the Cox proportional model, there was a $33 \%$ increased risk of death with increasing number of fibroblastic foci (hazard ratio 1.33, 95\% CI 0.72-2.46); however, this was not statistically significant (fig. 4b).

\section{Discussion}

These data show that high levels of $\alpha v \beta 6$ integrins are significantly associated with poor prognosis. There is also a trend towards worse prognosis with high levels of $\alpha$ SMA. However, there were no differences observed between samples with UIP or NSIP histology, although this study was not sufficiently powered to detect a difference between the two groups. Furthermore, there was no apparent relationship between the number of fibroblastic foci and mortality, consistent with previous reports [8]. This is the first study to demonstrate a tissue immunomarker in ILD with a significant association with the prognosis. A notable observation is that the median survival of patients with the highest expression of the $\alpha \mathrm{v} \beta 6$ integrin was only 25 months, which is comparable to the published survival data in IPF. This suggests that increased expression of the $\alpha v \beta 6$ integrin may represent a distinct endotype of progressive fibrotic ILD, and could be useful as a biomarker for disease progression and stratification of therapy.

There have been a number of studies that have tried to characterise molecular endotypes in ILD [7, 30-32]. The phenotype, which has been consistently shown to associate with poor survival on lung biopsy, is the presence of high levels of fibroblastic foci $[7,9,32]$. However, the assessment of fibroblastic foci relies on conventional histological techniques and does not reflect precise molecular endotyping. We did not find a significant association between either high numbers of fibroblastic foci or high levels of $\alpha \mathrm{SMA}$ and prognosis. These data are consistent with previous studies that have found only weak, nonsignificant correlations of active myofibroblasts with disease severity [31]. However, there was a trend towards a worse prognosis with higher levels of both fibroblastic foci and $\alpha \mathrm{SMA}$, which supports the notion that fibroblastic foci are an adverse feature in IPF biopsies, but larger studies may be required to accurately quantify this effect.

The role of $\alpha v \beta 6$ integrins in the pathogenesis of pulmonary fibrosis is well established and $\alpha v \beta 6$ immunostaining is increased in IPF, compared with control lung tissue [16-18]. Furthermore, a humanised anti- $\alpha v \beta 6$ monoclonal antibody, STX-100, is currently being evaluated in a phase 2 study. The association of $\alpha \mathrm{v} \beta 6$ integrin levels with prognosis of fibrotic ILDs has not been previously assessed. However, endotyping by tissue biopsy is an invasive procedure carrying significant mortality and morbidity irrespective of the mode of biopsy [33, 34]. The short-term mortality after lung biopsy in IPF is higher than that of other interstitial diseases [35]. Furthermore, there is an inherent sample bias in the surgical biopsies due to the patchy nature of the disease and assessment of a limited number of tissue sections within the whole lung biopsy. This is noted irrespective of the modality of the procedure undertaken to obtain the surgical lung biopsies (VATS, open-lung or transbronchial lung biopsies) [36]. An alternative to immunohistological assessment of lung biopsies could be through single photon emission computed tomography imaging. This technique is highly sensitive and able to globally quantify $\alpha v \beta 6$ integrin levels within the lung [37]. Therefore, molecular imaging of the $\alpha v \beta 6$ integrin may have advantages over immunohistochemistry as a prognostic biomarker in fibrotic ILDs. 
The strength of the study is the robust quantification methodology used for the tissue biomarkers. This study shows that operator analysis is similar to automated analysis. At present, the automated analysis is an expensive modality and often a research tool, whereas chromogenic staining is a well-established technique requiring only a standard microscope. In parallel with advances in cancer management, recent National Institute for Health and Care Excellence guidance in the UK proposes a multidisciplinary team (MDT) approach to IPF, and emphasises the importance of the pathologist [38]. The importance of these members of the MDT will become even greater if molecular phenotyping of lung biopsies follow the trajectory observed in lung cancer. However, interobserver variation and pathologist $\kappa$-scores are moderate even in the most robust of studies $[32,39]$, and the number of biopsies to be performed is likely to remain a small proportion of the total, due to the technical issues described previously. Therefore, exploring noninvasive strategies to define prognosis and response to therapy remains crucial.

The main limitation of the study lies in the small cohort size. Despite this the association of high levels of $\alpha v \beta 6$ integrin immunostaining with poor outcome was significant. Furthermore, a trend suggesting that high levels of $\alpha \mathrm{SMA}$ and high numbers of fibroblastic foci also lead to worse prognosis supported previous observations, and a larger cohort may aid confirmation. Whether a large prospective study defining the role of immunomarkers in IPF is a possibility, given the limitations of the diagnostic lung biopsy, in contrast with the strength of the diagnostic computed tomography scan, remains to be seen. Another limitation of the study is the absence of a control population; however, this study was designed to address the role of these molecules as prognostic, not diagnostic, biomarkers. Conventional controls for IPF immunohistochemistry are areas of normal lung taken from lung cancer patients undergoing lung resection and, clearly, this would not be an appropriate control for a prognostic study, and patients with truly normal lung do not undergo lung biopsy.

Despite the limitations of our study, the observations described are important because they highlight that molecular markers have potential to aid stratification and the determination of prognosis in IPF. Furthermore, given the importance of histopathology in the management of ILDs, it is vital that as much information can be gained from biopsies as possible, and molecular endotyping may increase their utility. Ultimately, this study demonstrates that high levels of the epithelium-restricted $\alpha v \beta 6$ integrin may identify an endotype of progressive fibrotic ILD with a poor prognosis, which may be appropriate for therapeutic targeting.

\section{Acknowledgements}

The authors thank South East Scotland SAHSC BioResource for access to the tissue and Alison John (University of Nottingham, Nottingham, UK) for help with the Nikon image analysis software.

\section{References}

1 Gribbin J, Hubbard RB, Le Jeune I, et al. Incidence and mortality of idiopathic pulmonary fibrosis and sarcoidosis in the UK. Thorax 2006; 61: 980-985.

2 Navaratnam V, Fleming KM, West J, et al. The rising incidence of idiopathic pulmonary fibrosis in the U.K. Thorax 2011; 66: 462-467.

3 Flaherty KR, Toews GB, Travis WD, et al. Clinical significance of histological classification of idiopathic interstitial pneumonia. Eur Respir J 2002; 19: 275-283.

4 Ryerson CJ, Urbania TH, Richeldi L, et al. Prevalence and prognosis of unclassifiable interstitial lung disease. Eur Respir J 2013; 42: 750-757.

5 Boon K, Bailey NW, Yang J, et al. Molecular phenotypes distinguish patients with relatively stable from progressive idiopathic pulmonary fibrosis (IPF). PLoS One 2009; 4: e5134.

6 Konishi K, Gibson KF, Lindell KO, et al. Gene expression profiles of acute exacerbations of idiopathic pulmonary fibrosis. Am J Respir Crit Care Med 2009; 180: 167-175.

7 King TE Jr, Schwarz MI, Brown K, et al. Idiopathic pulmonary fibrosis: relationship between histopathologic features and mortality. Am J Respir Crit Care Med 2001; 164: 1025-1032.

8 Hanak V, Ryu JH, de Carvalho E, et al. Profusion of fibroblast foci in patients with idiopathic pulmonary fibrosis does not predict outcome. Respir Med 2008; 102: 852-856.

9 Lee SH, Shim HS, Cho SH, et al. Prognostic factors for idiopathic pulmonary fibrosis: clinical, physiologic, pathologic, and molecular aspects. Sarcoidosis Vasc Diffuse Lung Dis 2011; 28: 102-112.

10 Ohnishi H, Yokoyama A, Kondo K, et al. Comparative study of KL-6, surfactant protein-A, surfactant protein-D, and monocyte chemoattractant protein-1 as serum markers for interstitial lung diseases. Am J Respir Crit Care Med 2002; 165: 378-381.

11 Vuga LJ, Tedrow JR, Pandit KV, et al. C-X-C motif chemokine 13 (CXCL13) is a prognostic biomarker of idiopathic pulmonary fibrosis. Am J Respir Crit Care Med 2014; 189: 966-974.

12 Prasse A, Probst C, Bargagli E, et al. Serum CC-chemokine ligand 18 concentration predicts outcome in idiopathic pulmonary fibrosis. Am J Respir Crit Care Med 2009; 179: 717-723.

13 DePianto DJ, Chandriani S, Abbas AR, et al. Heterogeneous gene expression signatures correspond to distinct lung pathologies and biomarkers of disease severity in idiopathic pulmonary fibrosis. Thorax 2015; 70: 48-56.

14 Rosas IO, Richards TJ, Konishi K, et al. MMP1 and MMP7 as potential peripheral blood biomarkers in idiopathic pulmonary fibrosis. PLoS Med 2008; 5: e93.

15 Munger JS, Huang X, Kawakatsu H, et al. The integrin $\alpha v \beta 6$ binds and activates latent TGF $\beta 1$ : a mechanism for regulating pulmonary inflammation and fibrosis. Cell 1999; 96: 319-328. 

LPA2 receptor and the small G protein $\mathrm{G \alpha}$. Am I Pathol 2009; 174: 1264-1279.

Horan GS, Wood S, Ona V, et al. Partial inhibition of integrin $\alpha_{\mathrm{v}} \beta 6$ prevents pulmonary fibrosis without exacerbating inflammation. Am J Respir Crit Care Med 2008; 177: 56-65.

19 Kobayashi H, Gabazza EC, Taguchi O, et al. Protein C anticoagulant system in patients with interstitial lung disease. Am J Respir Crit Care Med 1998; 157: 1850-1854.

20 Nogee LM, Dunbar AE III, Wert S, et al. Mutations in the surfactant protein C gene associated with interstitial lung disease. Chest 2002; 121: Suppl., 20S-21S.

21 Scotton CJ, Chambers RC. Molecular targets in pulmonary fibrosis: the myofibroblast in focus. Chest 2007; 132: $1311-1321$.

22 Yang J, Dai C, Liu Y. Hepatocyte growth factor gene therapy and angiotensin II blockade synergistically attenuate renal interstitial fibrosis in mice. J Am Soc Nephrol 2002; 13: 2464-2477.

23 Marchand-Adam S, Fabre A, Mailleux AA, et al. Defect of pro-hepatocyte growth factor activation by fibroblasts in idiopathic pulmonary fibrosis. Am J Respir Crit Care Med 2006; 174: 58-66.

24 Kaarteenaho-Wiik R, Tani T, Sormunen R, et al. Tenascin immunoreactivity as a prognostic marker in usual interstitial pneumonia. Am J Respir Crit Care Med 1996; 154: 511-518.

25 Kuhn C, Mason RJ. Immunolocalization of SPARC, tenascin, and thrombospondin in pulmonary fibrosis. Am J Pathol 1995; 147: 1759-1769.

26 Weinreb PH, Simon KJ, Rayhorn P, et al. Function-blocking integrin alphavbeta6 monoclonal antibodies: distinct ligand-mimetic and nonligand-mimetic classes. J Biol Chem 2004; 279: 17875-17887.

27 Idiopathic pulmonary fibrosis: diagnosis and treatment. International consensus statement. American Thoracic Society (ATS), and the European Respiratory Society (ERS). Am J Respir Crit Care Med 2000; 161: 646-664.

28 Fisher ER, Anderson S, Dean S, et al. Solving the dilemma of the immunohistochemical and other methods used for scoring estrogen receptor and progesterone receptor in patients with invasive breast carcinoma. Cancer 2005; 103: $164-173$.

29 Katzenstein AL, Myers JL. Idiopathic pulmonary fibrosis: clinical relevance of pathologic classification. Am J Respir Crit Care Med 1998; 157: 1301-1315.

30 Harada T, Nabeshima K, Hamasaki M, et al. Epithelial-mesenchymal transition in human lungs with usual interstitial pneumonia: quantitative immunohistochemistry. Pathol Int 2010; 60: 14-21.

31 Lomas NJ, Watts KL, Akram KM, et al. Idiopathic pulmonary fibrosis: immunohistochemical analysis provides fresh insights into lung tissue remodelling with implications for novel prognostic markers. Int J Clin Exp Pathol 2012; 5: 58-71.

32 Nicholson AG, Fulford LG, Colby TV, et al. The relationship between individual histologic features and disease progression in idiopathic pulmonary fibrosis. Am J Respir Crit Care Med 2002; 166: 173-177.

33 Carnochan FM, Walker WS, Cameron EW. Efficacy of video assisted thoracoscopic lung biopsy: an historical comparison with open lung biopsy. Thorax 1994; 49: 361-363.

34 Miller JD, Urschel JD, Cox G, et al. A randomized, controlled trial comparing thoracoscopy and limited thoracotomy for lung biopsy in interstitial lung disease. Ann Thorac Surg 2000; 70: 1647-1650.

35 Utz JP, Ryu JH, Douglas WW, et al. High short-term mortality following lung biopsy for usual interstitial pneumonia. Eur Respir J 2001; 17: 175-179.

36 Flaherty KR, Travis WD, Colby TV, et al. Histopathologic variability in usual and nonspecific interstitial pneumonias. Am J Respir Crit Care Med 2001; 164: 1722-1727.

37 John AE, Luckett JC, Tatler AL, et al. Preclinical SPECT/CT imaging of alphavbeta6 integrins for molecular stratification of idiopathic pulmonary fibrosis. J Nucl Med 2013; 54: 2146-2152.

38 National Institute for Health and Clinical Excellence. Idiopathic pulmonary fibrosis: The diagnosis and management of suspected idiopathic pulmonary fibrosis. www.nice.org.uk/guidance/cg163 Date last updated: June 2013.

39 Walker RA. Quantification of immunohistochemistry - issues concerning methods, utility and semiquantitative assessment I. Histopathology 2006; 49: 406-410. 